\title{
Zur methodischen therapeutisch-klinischen Forschung der «Ecole de Paris» (1800-1850)
}

\author{
Von Eduard-Rudolf Müllener, Bern
}

Die Medizin der sogenannten Pariser klinischen Schule in der ersten Hälfte des 19. Jahrhunderts war nach AcKerknecht bereits «une médecine moderne, sans être encore $l a$ médecine moderne ${ }^{1}{ }^{1}$ Der mit dieser prägnanten Formulierung umschriebene Pioniergeist der damaligen Pariser Medizin wurde verschiedentlich im Hinblick auf die allgemeine Diagnostik sowie diverse Spezialgebiete (z.B. Gerichtsmedizin, Venerologie, Orthopädie u.a.m.) dargestellt ${ }^{2}$; die Therapie der Pariser Kliniker fand ebenfalls ihre Bearbeitung ${ }^{3}$.Hingegen fehlt bis dahin eine nähere Würdigung des Umstands, daß auch die gerade in unserer Zeit stark aufstrebende methodische klinisch-therapeutische Forschung im nachrevolutionären Paris ihren Ausgang nahm; einigen Aspekten jener ersten deutlichen Ansätze zu einer solchen Betrachtungsweise sei diese kurze Skizze gewidmet.

Die erwähnte Forschung, deren modernste Grundlagen vor allem MARTINI herausarbeitete und vervollkommnete, befaßt sich mit der Überprüfung von Behandlungsverfahren am kranken Menschen, wobei eine bestimmte Methodik möglichst viele Irrtümer, Zufälle und fremde Einflüsse ausschalten soll. Martinis 1932 erstmals erschienenes Buch Methodenlehre der therapeutisch-klinischen Forschung hebt in diesem Zusammenhang folgenden Punkt als besonders wichtig heraus: die methodische therapeutische Forschung unterscheidet sich von der bloßen therapeutischen Erfahrung am einzelnen Kranken (die nach Martini der sogenannten naiven Erfahrung der Philosophie gleichgesetzt werden kann) prinzipiell durch das Hineintragen des experimentellen Gesichtspunkts in die Therapie ${ }^{4}$. Nun läßt sich

1 E. H.Ackerknecht, La médecine à Paris entre 1800 et 1850. Les conférences du Palais de la Découverte Série D, Nr. 58 (Conférence faite au Palais de la Découverte le 19 avril 1958), S.5.

2 Siehe Anmerkung 1 sowie z.B. G.BAss, Die Gerichtsmedizin als Spezialfach in Paris von 1800-1850, Zürich 1964; P. WeIdmanN, Die Venerologie in Paris von 1800-1850, Zürich 1965 ; E.A. Häfliger, Die Orthopädie in Paris von 1800-1850, Zürich 1965.

3 E.H.Ackerknecht, Die Therapie der Pariser Kliniker zwischen 1795 und 1840, Gesnerus 15 (1958) 151-163.

4 P.MaRtini, Methodenlehre der therapeutisch-klinischen Forschung, 3. Auflage, Berlin/ Göttingen/Heidelberg 1953, S. $6 \mathrm{ff}$. 
das ideale Experiment im Sinne der Naturwissenschaften unter bestimmten vom Experimentator gewählten und von ihm je nach den Erfordernissen variierbaren Bedingungen beliebig oft wiederholen; die dabei beobachteten Phänomene sind meß- sowie vergleichbar, und ihre Anzahl ist praktisch unbegrenzt.

Da aber der erkrankte Mensch von einer menschenwürdigen Medizin nie gänzlich einem Objekt der Naturwissenschaft gleichgesetzt werden kann, sieht sich die experimentelle therapeutische Forschung von allem Anfang an gezwungen, verschiedene deutliche Abstriche an diesen Idealforderungen hinzunehmen: der Arzt kann die Voraussetzungen des Versuches nicht selber bestimmen oder gar verändern, sondern muß sie so akzeptieren, wie die Krankheit sie darbietet. Es ist ihm ferner meist versagt, die Versuche nach Belieben zu wiederholen oder ihre Anzahl ins Unbegrenzte zu steigern, und er hat relativ wenig exakte Meßmöglichkeiten. Diesen Umständen entspringt nicht zuletzt die Schwierigkeit, überhaupt eine einigermaßen gültige Vergleichsbasis zu finden. Angesichts solcher Einschränkungen empfiehlt Martini als Hauptmethode der systematischen therapeutischen Forschung (vor allem bei akuten Krankheiten) den sogenannten kollektiven therapeutischen Vergleich, d.h. den Vergleich zwischen zwei (oder mehr) möglichst großen und homogenen Kollektiven von Kranken, die verschiedene Behandlungsweisen erfahren; die Ergebnisse sollen mit Hilfe der Wahrscheinlichkeitsrechnung gesichert werden ${ }^{5}$. Von den mit diesem Grundprinzip verbundenen vielfältigen Problemen seien hier nur einige wenige noch kurz herausgehoben, wie z.B. die nötige Vorausplanung des Versuches, die Fragen der genügenden Homogenität und Konstanthaltung der gesamten Versuchsbedingungen oder die Idee der Ausschaltung möglicher psychischer Einwirkungen durch Placeboabgabe (einfacher und doppelter Blindversuch); die jeweils optimalen Lösungen bestimmter Aufgaben werden gerade heute wieder häufig diskutiert und sind vielfach noch umstritten.

Erste deutliche theoretische und auch praktische Ansätze zu einer ähnlichen Betrachtung der Therapiefrage lassen sich nun in der nachrevolutionären Pariser Medizin nachweisen, die bekanntlich vom methodischen Dogmatismus (oder Rationalismus) des 18. Jahrhunderts abrückte und zu einem skeptischen Empirismus gelangte. Ein Zeitgenosse wählte für diese Rich-

5 1.c., S. 14. Für chronische Krankheiten stellt er ebenda den Verlaufsvergleich in den Vordergrund. 
tung die glückliche Bezeichnung «empirisme raisonné», ${ }^{6}$ welche deutlich die Verschmelzung der alten Gegensätze Rationalismus und Empirismus zu etwas wirklich Neuem zeigt, nämlich zu einer kontrollierten Empirie ${ }^{\text {. }}$ Den Bemühungen um eine möglichst systematische Kontrolle der bei der Behandlung von Kranken gemachten Erfahrungen entspringen nun die ersten Keime einer methodischen klinisch-therapeutischen Forschung. Ihr theoretisches Fundament lieferte der Ideologe Cabanis, indem er 1798 die Unmöglichkeit darlegte, Behandlungsverfahren aus rein theoretischen Erwägungen über das Wesen der Krankheit abzuleiten; er hielt auch die Einsicht in den Zusammenhang zwischen dem Krankheitsgeschehen und der Behandlung für überflüssig - die Therapie soll allein auf dem Wege der systematischen Erfahrung eruiert werden ${ }^{8}$. Die Grundzüge einer solchen Systematik sowie ihre erste Anwendung in der Praxis verdanken wir Pinel, der in seinem Ringen um die Verbesserung der Nosologie zum Postulat einer gewissen Spezifität der Krankheiten gelangte. Und nur unter dieser Voraussetzung, der Annahme von einigermaßen feststehenden und konstant bleibenden Krankheitsbildern oder -einheiten, ist eine systematische therapeutische Untersuchung überhaupt möglich. Pinel suchte den spezifischen Charakter der Krankheiten durch die Hervorhebung ihrer typischen Leitsymptome herauszuschälen und verwies die übrigen Umstände wie Alter, Geśchlecht, Konstitution, Klima, Jahreszeit u.ä.m. (die im 18. Jahrhundert offensichtlich stark überschätzt wurden) als bloß modifizierende Faktoren auf den zweiten Platz ${ }^{9}$. Derselbe Gesichtspunkt findet Anwendung in der Psychiatrie, wo er zweifellos seinen «genres de maladie» (manie, mélancholie, démence, idiotisme; gelegentlich mit weiteren Unterteilungen in z.B. mélancholie avec délire sur un seul objet, mélancholie avec penchant au suicide u.ä.m.) eine gewisse Eigenständigkeit zuerkennt. Gestützt auf

${ }^{6}$ L. Delasiauve, Gazettehebdomadaire de médecine et de chirurgie 2 (1855) 454-455, 455 (Besprechung der 5 . Auflage von A.Trousseau und H. Pidoux, Traité de thérapeutique et de matière médicale, Paris 1855).

7 Schon die alexandrinischen Empiriker scheinen erkannt zu haben, daß mit der Zahl der Beobachtungen der Sicherheitsgrad der Erfahrung wächst (n. B. Milt, Empirie und das statistisch fundierte biologisch-medizinische Denken in der Geschichte, Gesnerus 13 (1956) 1-28, 3). Die Pariser Kliniker wurden in dieser Hinsicht durch den Umstand begünstigt, daß sich zu ihrer Zeit die Krankenbettmedizin in die Spitalmedizin mit viel größeren Patientenzahlen umwandelte.

8 P.J.G. Cabanis, Du degré de certitude dans la médecine, 2. Auflage, Paris 1803, S. $60 \mathrm{ff}$.

9 Siehe z.B. Рh. Pinel, Classification des maladies internes, Dictionnaire des sciences médicales 5 (1813) 267-287, 279. 
diese Annahme macht er sich dann an die schwierige Aufgabe, die Heilungschancen der Irren überhaupt und jedes «genres» zu untersuchen; seine entsprechende Schrift aus dem Jahre 1807, die über das Schicksal von insgesamt 517 Kranken während rund 4. Jahren berichtet, kann als erster einigermaßen kontrollierter therapeutischer Versuch gelten und enthält gleichzeitig die wichtigsten theoretischen Grundsätze der methodischen therapeutisch-klinischen Forschung. "Une expérience» schreibt Pinel, «pour être authentique et concluante, et servir de fondement solide à une méthode quelconque de traitement, doit être faite sur un grand nombre de malades, asservis à des règles générales et dirigés suivant un ordre déterminé. Elle doit être aussi établie sur une succession régulière d'observations constatées avec un soin extrême et répétées pendant un certain nombre d'années avec une sorte de conformité. Enfin elle doit rapporter également les évènements favorables comme ceux qui sont contraires, assigner leurs nombres respectifs, et instruire autant par les uns que par les autres. C'est assez dire qu'elle doit être fondée sur la théorie des probabilités... sur laquelle doivent désormais porter les méthodes de traitement des maladies, si on veut les établir sur un fondement solide». ${ }^{10}$ Weiter unten heißt es dann noch: «...on se dirige par une comparaison continuelle des rapports obtenus entre le nombre des guérisons et celui des admissions, soit avec les rapports obtenus antérieurement dans le même lieu, soit avec ceux des autres hospices tenus avec régularité».11 Hier ist in wenigen Worten fast das ganze Programm der methodischen klinisch-therapeutischen Forschung umrissen: große Anzahl von Kranken, Konstanthaltung der Bedingungen, genaue Beobachtung, absolute Aufrichtigkeit (Angabe der nicht geheilten Fälle), Vergleich der Ergebnisse und Bearbeitung der Resultate mittels der Wahrscheinlichkeitsrechnung. Zum letzten Punkt bemerkt Pinel allerdings: «...la probabilité d'un évènement se mesure par une fraction dont le numérateur est le nombre des cas favorables, et le dénominateur, le nombre de tous les cas possibles, favorables ou contraires "; ${ }^{12}$ was zeigt, daß Pinel unter Wahrscheinlichkeit einfach den ausgezählten Anteil von Heilungen versteht.

10 Ph. Pinel, Résultats d'observations et construction des tables pour servir à déterminer le degré de probabilité de la guérison des aliénées, Mémoires de la classe des sciences mathématiques et physiques de l'Institut National de France 8 (1807) 169-205, 169.

11 1.c., S.187/188.

12 1.c., S. 172. 
Diese von Pinel empfohlene Häufigkeitsstatistik oder das Zählen schlechthin erfuhr dann bekanntlich in LouIs' "méthode numérique» eine weitere Verfeinerung und großzügigere Anwendung. Louis präzisierte auch die theoretischen Grundlagen der methodischen klinisch-therapeutischen Forschung, indem er z.B. unter anderem den Leerversuch bzw. eine Kontrollgruppe unbehandelter Kranker forderte: «...il est nécessaire de comparer entre eux un assez grand nombre de cas d'une même affection, au même degré; les uns relatifs à des sujets dont la maladie aura été abandonnée à elle-même; les autres relatifs à des individus auxquels tels ou tels médicaments auront été administrés ». ${ }^{13}$ Die praktische Durchführung dieser theoretischen Forderung blieb allerdings einstweilen aus; Louis selber weist auf die Schwierigkeit ihrer Verwirklichung hin, indem er schreibt: «...le péril (sc. des maladies graves) ...ne permet guère de rester simple spectateur».14 Eine Kontrollgruppe fehlt denn auch in seinen Untersuchungen über die Wirksamkeit des Aderlasses, die - im Rahmen des damals überhaupt Möglichen gesehen - einen ersten Höhepunkt der systematischen therapeutischen Forschung bedeuten; als Hauptkriterien des therapeutischen Effekts betrachtet er die Dauer und den Verlauf der Krankheit bei in verschiedenen Zeitpunkten der Erkrankung zur Ader gelassenen Patientengruppen; die zahlenmäßig erfaßten und in synoptischen Tafeln dargestellten Ergebnisse leiten ihn zur Schlußfolgerung, daß der Aderlaß entzündliche Leiden nur wenig zu beeinflussen vermag ${ }^{15}$. Diese erste methodisch fundierte, aber noch sehr vorsichtig gefaßte Kritik an einer seit der Antike praktizierten Behandlungsform erschien Louis selber dermaßen revolutionär, daß er sie mit den Worten beginnt: «Le résultat de mes recherches sur les effets de la saignée dans les inflammations est si peu d'accord avec l'opinion commune, que ce n'est pas sans une sorte d'hésitation que je me décide à les publier ${ }^{16}{ }^{16}$ Das Fehlen einer Kontrollgruppe unbehandelter Kranker wurde ihm auch prompt von BouILLAUD vorgeworfen: «...pour connaitre la part que peut prendre la saignée à la guérison, il faudrait comparer les cas où ce traitement a été mis en usage, à d'autres cas dans lesquels on ne l'aurait pas em-

13 P.-Сн.-A.Louis, Recherches anatomiques, pathologiques et thérapeutiques sur la maladie connue sous les noms de fièvre typhoide, etc., 2. Auflage, Band 2, S.379, Paris 1841.

14 1.c., S. 380.

15 P.-Сн.-A.Louis, Recherches sur les effets de la saignée dans quelques maladies inflammatoires, Paris 1835, S. 31 .

16 1.c., S. 7. 
ployé ». ${ }^{17}$ Dies hindert Bouillaud allerdings nicht, mit einer Erfolgsstatistik - ohne Kontrollgruppe - die Überlegenheit seiner «saignée coup sur coup» zu beweisen ${ }^{18}$ ! Aus seinem Werk geht (wenn auch nicht explicite) recht deutlich hervor, daß er von vornherein die «saignée coup sur coup» für absolut zuverlässig hielt und im übrigen vom Gedanken besessen war, die Behandlungsergebnisse von Louis zu verbessern, welche er auch - ziemlich ungerechtfertigterweise, da Louis' Zielsetzung eine andere war - als Vergleichsbasis heranzog; unter solchen Auspizien rückt natürlich der Gedanke an einen Leerversuch völlig in den Hintergrund.

Gerade die ersten tastenden Ansätze zu einer methodischen klinisch-therapeutischen Forschung lassen also deutlich die Schwierigkeiten einer praktischen Anwendung der theoretisch bereits recht gut formulierten Methodik erkennen. Viele Zeitgenossen übten denn auch an der eben im Entstehen begriffenen neuartigen Untersuchungstechnik eine wegen deren Unzulänglichkeiten zum Teil gerechtfertigte Kritik, aber ebenso häufig eine völlig unsinnige Polemik, welche von vornherein jede Möglichkeit oder Nützlichkeit solcher Studien überhaupt verneinte. Darauf soll hier nicht eingegangen werden; es genüge der Hinweis, daß die Methode an sich bis zur Mitte des vergangenen Jahrhunderts schließlich doch im großen und ganzen als brauchbares Forschungsinstrument anerkannt wurde - eine Tatsache, die z. B. von Broca in einem das Problem berührenden Artikel durch folgenden lapidaren Satz festgehalten wird: «Je suis obligé de traiter des malades, et je ne serais pas fâché... de savoir quelle est la méthode qui réussit le plus souvent ». ${ }^{19}$ Für dieselbe Tatsache spricht auch der Umstand, daß viele bekannte Pariser Ärzte jener Epoche therapeutische Forschung mit mehr oder weniger ausgeprägtem methodischem Einschlag zumindest versuchten; allerdings fällt es im Rückblick recht schwer, den Punkt zu bestimmen, von welchem an eine therapeutische Studie «systematisch» genannt werden soll, da man mit Rücksicht auf die damalige Gesamtlage der Medizin die Anforderungen nicht zu sehr in die Höhe schrauben darf, und da ein fließender Übergang mit vielen Stufen vorliegt zwischen z.B. der bloßen Errechnung der Mortalitätsziffer eines Eingriffs (welcher aber auch schon eine ge-

17 J.BoullLaud, Essai sur la philosophie médicale et sur les généralités de la clinique médicale, Paris 1836, S. 335.

18 1.c., S. $345 \mathrm{ff}$.

19 P.Broca, Du degré de l'utilité de la statistique, Le Moniteur des Hôpitaux, $1^{\mathrm{e}}$ sér., 5 (1857) 33-36, 41-47, 33. 
wisse primitive Systematik innewohnt) und dem Idealtyp der möglichst perfekt a priori organisierten und mit methodischer Strenge durchgeführten Untersuchung. Zur erstgenannten Art gehört z.B. die Mortalitätsstatistik von Malgaigne, der auf Grund von insgesamt 789 Fällen von Amputation eine Sterblichkeit von $40 \%$ bei Absetzung des Gliedes wegen Krankheit und von $50 \%$ bei derselben Operation nach Verletzung errechnet, wozu er schreibt: «Der Eindruck, den meine Arbeit auf mich selbst gemacht hat, war betrübend. Die Fortschritte der neueren Chirurgie erscheinen viel weniger glänzend, wenn man sie nach jenen schauerlichen Sterblichkeitslisten bemißt $» .^{20}$ Etwa in derselben Ebene liegen die Untersuchungen über die Streitfrage der Zertrümmerung oder Exzision von Blasensteinen; Civiale sammelte zu diesem Problem an die 6000 Fälle; bei über 5000 Steinschnitten errechnet er eine Mortalität von $1: 5$, bei rund 250 Steinzertrümmerungen eine solche von $1: 40^{21}$. Velpeau, der die Lithotomie der Lithotritie vorzieht, antwortet darauf mit einer kleinen Statistik von 24 Steinzertrümmerungen, wobei 4 Patienten an dem Eingriff starben, was eine Mortalität von $1: 6$ ergibt ${ }^{22}$. Diese Art von Untersuchungen und vor allem Gegenüberstellungen mag primitiv erscheinen - nichtsdestoweniger sind es erste Ansätze zu einer systematischeren Betrachtung von Therapieverfahren mittels Häufigkeitszählung und Vergleich. Das weitere Schicksal der Methode sei hier am typischen Beispiel der Diskussion über die Typhus -Therapie in der "Académie Royale de Médecine» aus den Jahren 1836/37 noch etwas ausführlicher erörtert. Den Anstoß dazu gab eine von De Larroque der Akademie eingereichte Arbeit, worin er mit seinen Mitarbeitern folgenden ursprünglichen Plan zur Ausführung bringen wollte: «Ils voulaient arriver à traiter d'abord 100 malades par la méthode proposée par M. de Larroque (sc. purgatifs), puis 100 autres par la méthode antiphlogistique, 100 autres enfin par une méthode à peu près expectante $» .^{23}$ Hier also ein methodisch nahezu perfekter theoretischer Ansatz, in welchem auch der Leerversuch zumindest angedeutet ist (méthode expectante). Leider erwies sich - aus

20 Zit. nach C.A. Wunderlich, Proben von Malgaigne's Arbeiten über chirurgische Statistik, Archiv für physiologische Heilkunde 1 (1842) 477-491, 491.

21 J. Civiale: Recherches de statistique sur l'affection calculeuse, Comptes rendus hebdomadaires des séances de l'Académie des Sciences 1 (1835) 167-177, 172.

22 A. Velpeau, Taille et lithotritie, Gazette médicale de Paris $3^{e}$ sér., 2 (1847) 833-834, 834.

${ }^{23}$ B. De LaRroque, Observations sur le traitement de la fièvre typhoide par les purgatifs, Bulletin de l'Academie royale de médecine 1 (1836/37) 482-502, 517-526, 529-542, 549$558,588-602,483$. 
nicht näher geschilderten Gründen - seine Umsetzung in die Praxis als unmöglich, und de Larroque mußte sich damit begnügen, 100 Typhuskranke ausschließlich purgativ zu behandeln. Dies tat er in einer offensichtlich für jene Zeit aufsehenerregend gründlichen Art: «...généralisant... l'emploi de la méthode évacuante, et ne la restreignant plus seulement à certains cas; poursuivant par elle pendant toute la durée de l'état pyrétrique; ne tenant compte ni de la diversité des symptômes, ni des lésions, ni de diversité même des prédispositions individuelles ${ }^{24}$... le traitement est un comme est une la nature de la maladie ».25 Hier finden wir Pinels Idee der Spezifität von Krankheit (die seither vor allem durch Louis' Untersuchungen über Tuberkulose und Typhus erhärtet worden war) und Heilmethode in aller Schärfe formuliert wieder. Wie aus der Diskussion der «Académie» über deLarroques Untersuchung hervorgeht, stieß seine strikte Durchsetzung eines einzigen Behandlungsprinzips bei sämtlichen Patienten ohne Rücksicht auf Nuancen im Krankheitsverlauf oder individuelle Momente u.ä.m. - ein Verfahren ganz im Sinne der methodischen therapeutischen Forschung - vor allem bei den konservativen Ärzten auf heftigen Widerstand. Von seinen 100 Kranken starben nur 10, was als sehr gutes Ergebnis gelten konnte, indem damals (vor allem seit den Untersuchungen von Louis) die Typhusmortalität allgemein auf $1 / 3$ geschätzt wurde. Gewissermaßen als Ersatz für die ursprünglich geplante große Untersuchung von deLarroque trägt dann ANDral aus eigenen Erfahrungen sowie jenen von de Larroque, Louis und PiÉdagnel 372 Typhusfälle zusammen, die verschiedene Behandlungen durchmachten; er kommt zu folgenden Sterblichkeitsraten (wobei allerdings die einzelnen Gruppen sehr stark differierende Krankenzahlen aufweisen) : bei Therapie mit «simple délayans » gleich Null, mit «évacuans seuls» gleich 1/7 (was etwa dem Mortalitätsdurchschnitt der Gesamtzahl gleichkommt), mit «émissions sanguines peu abondantes» gleich 1/4 und mit Aderlaß plus Evakuans gleich 1/3. Zur ganzen Sachlage bemerkt Andral mit Recht: «Avec ces résultats, instituerons-nous la science? Non... parce que, dans les faits qui ont produit chacun d'eux, il n'y a pas de parité suffisante à établir ni quant au nombre ni quant à la nature $\gg{ }^{26}$ Immerhin läßt sich aus den Ergebnissen bei aller gebotenen Vorsicht der Schluß ziehen, daß eine möglichst schonende Behandlung (wie sie bereits Pinel als «traite-

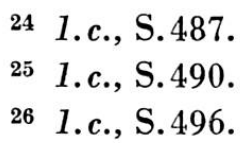


ment expectatif» vertreten hatte) am Platz sein dürfte. Louis kommt 1841 in der zweiten Auflage seines Werkes über den Typhus in der Therapiefrage trotz einiger zusätzlicher Untersuchungen auch nicht weiter als bis zu dieser allgemeinen Einsicht ${ }^{27}$; er weist aber zumindest genau auf die Hauptschwierigkeit der damaligen therapeutischen Situation hin, nämlich auf das Problem, wie man die von der pathologischen Anatomie festgestellten Zerstörungen behandeln soll: «...l'altération plus ou moins marquée de la structure de l'intestin, c'est un symptôme formidable; quel moyen lui opposer? Sur ce point comme sur tant d'autres, l'expérience est muette».28 Die Ohnmacht aller bekannten Behandlungsmöglichkeiten trug zweifellos das ihre bei zum Scheitern vieler Versuche mit methodischem Einschlag.

Hatte zuerst die Skepsis Ansätze in Richtung einer systematischen therapeutisch-klinischen Forschung gezeitigt, so brachte diese wiederum wenig ermutigende Ergebnisse und neue Skepsis hervor, was zu ihrem Niedergang nach 1850 beigetragen haben mag. Der anschließend einsetzende Aufstieg von Histopathologie und Bakteriologie eröffnete die Aussicht, primäre Ursachen der Erkrankungen zu eruieren und die Behandlung danach zu richten, was den Verzicht auf die kontrollierte therapeutische Empirie weiterhin erleichterte. Diese nahm erst in unserem Jahrhundert in verbesserter Form wieder einen starken Aufschwung. Ein Rückblick vom heutigen Standpunkt läßt erkennen, daß die von der Ecole de Paris entwickelten theoretischen Grundsätze einer methodischen therapeutischen Forschung prinzipiell richtig waren und im Keime fast alle Postulate des modernen Wissenschaftszweiges enthielten, so z.B. als Hauptverfahren den vorausgeplanten und kollektiven therapeutischen Vergleich mit zahlenmäßiger Auswertung der Ergebnisse. Was vor mehr als hundert Jahren noch fehlte, war einmal die Anwendung der statistischen Methodenlehre, deren eigentliche Entwicklung und Übertragung auf Biologie und Medizin erst in der zweiten Hälfte des 19. Jahrhunderts erfolgte, und zum andern der Blindversuch mittels Placebo; das Placeboproblem war zwar damals in einfacher Form keineswegs unbekannt ${ }^{29}$, doch spielten psychische Einflüsse bei der großen Zahl von wirklich schweren körperlichen Krankheiten eine so ver-

27 Siehe Anmerkung 13, z.B. S. 426, 436.

28 1.c., S. 496.

29 Vgl. dazu z.B. A.F. Сномец, Eléments de pathologie générale, 4. Auflage, Paris 1856, S. 576: «L'influence de l'imagination sur l'organisme est telle, que dans un certain nombre de cas, et chez certains individus, la pensée que tel remède devait produire tel effet a suffi pour que l'effet ait eu lieu.» Сномец gibt auch einige praktische Beispiele. 
schwindend kleine Rolle, daß offensichtlich ihre (auch nur theoretische) Einbeziehung in die Versuchsanordnung völlig fern lag. Die praktische Verwirklichung der von ihnen aufgestellten Grundsätze blieb allerdings bei den Pariser Klinikern fragmentarisch, und auch die einzelnen Fragmente weisen noch große Unzulänglichkeiten auf, wie z.B. Fehlen des Vergleichs überhaupt, unbedenkliches Heranziehen der Ergebnisse anderer Ärzte als Vergleichsbasis, von Gruppe zu Gruppe stark differierende Fallzahlen u. ä.m.

Die Hauptauswirkung der theoretisch zwar recht gut fundierten, aber noch sehr unvollkommen in die Praxis umgesetzten methodischen therapeutisch-klinischen Forschung der Ecole de Paris bestand zweifellos in einer Unterminierung der Traditionsgläubigkeit (z. B. Zweifel an der Wirksamkeit des Aderlasses) und Bloßstellung der damaligen therapeutischen Ohnmacht der Medizin ${ }^{30}$ - also in einer Ernüchterung, die ihr einerseits viele Feinde zuzog, andrerseits aber als heilsam und fruchtbar betrachtet werden muß im Sinne von Lotzes Worten: «Zwischen den Bedürfnissen des Gemütes und den Ergebnissen menschlicher Wissenschaft besteht ein alter, nie geschlichteter Zwist. Jene hohen Träume des Herzens aufzugeben, die den Zusammenhang der Welt anders und schöner gestaltet wissen möchte, als der unbefangene Blick der Beobachtung ihn zu sehen vermag, diese Entsagung ist zu allen Zeiten als der Anfang jeglicher Einsicht gefordert worden.»

${ }^{30}$ Vgl. dazu Chomel, 1.c., S.588: «...la mission évidente de la méthode numérique est de détruire les illusions». 